Results: Synovitis was found in MTP joints in $41(14.6 \%)$ of 280 toes. The incidence rates of synovitis in the deformity group, the bone erosion group, and the normal group were $27.3 \%, 13.1 \%$, and $6.7 \%$, respectively. Synovitis was found in $21.7 \%$ of patients on therapy with biologic products and in $38.3 \%$ of those without such therapy. There were no significant differences in the mean duration of the disease, visual analogue scale score, erythrocyte sedimentation rate, matrix metallopeptidase 3 level, or health assessment questionnaire score among the 3 groups.

Conclusions: Synovitis was also found in patients who showed no changes on imaging of the toes. Synovitis persisted in some patients even after establishment of toe deformity. Drug therapy, intensification of conservative therapy, and synovectomy should be considered to prevent further deformation.

References:

[1] Wakefield RJ. Ann Rheum Dis 63; 382-5: 2004

[2] Brown AK. Arthtitis Rheum 58(10); 2958-67: 2008

Acknowledgements: The authors wish to acknowledge Miss Sasaki, for her help in interpreting the significance of the results of this study.

Disclosure of Interest: None declared

DOI: 10.1136/annrheumdis-2017-eular.1244

\title{
AB1064 CARPAL TUNNEL BIOPSY AND BONE SCINTIGRAPHY USING THE TECHNETIUM-3,3-DIPHOSPHONO-1,2- PROPANODICARBOXYLIC ACID (99M) (TC-DPD) TRACER CAN IDENTIFY CLINICALLY SILENT CARDIAC AMYLOIDOSIS AT A POTENTIALLY TREATABLE STAGE
}

T.A.-B. Youngstein ${ }^{1}$, S. Tsamados $^{2}$, J.A. Gilbertson ${ }^{1}$, L. Smith ${ }^{1}$, D. Hutt ${ }^{1}$, T. Lane ${ }^{1}$, D. Rowczenio ${ }^{1}$, T. Rezk ${ }^{1}$, C.C. Quarta ${ }^{1}$, R. Manwani ${ }^{1}$, C. Whelan ${ }^{1}$, M. Fontana ${ }^{1}$, H.J. Lachmann ${ }^{1}$, J.D. Gillmore ${ }^{1}$, N. Goddard ${ }^{2}$, P.N. Hawkins ${ }^{1}$. ${ }^{1}$ National Amyloidosis Centre, UCL Division of Medicine; ${ }^{2}$ Department of Orthopaedics, Royal Free London NHS Foundation Trust, London, United Kingdom

Background: Carpal tunnel syndrome (CTS) is the only known early clinical manifestation of wild-type transthyretin amyloidosis (ATTRwt; formerly known as senile systemic amyloidosis) which causes an amyloid cardiomyopathy. At the UK National Amyloidosis Centre 98\% of those with proven ATTRwt have evidence of median nerve entrapment on neurophysiological studies and $48 \%$ have a history of carpal tunnel decompression as much as 12 years prior to heart failure symptoms. ATTRwt is diagnosed in approximately 150 individuals in the UK each year although post-mortem studies suggest presence of ATTwt amyloid deposits $30 \%$ of males over 80 years $(\mathrm{yrs})^{1}$. A novel bone tracer, Technetium-3,3diphosphono-1,2-propanodicarboxylic acid (99m) (Tc-DPD) largely abrogates the need for cardiac biopsy, identifying cardiac ATTR amyloidosis with a sensitivity of $98 \%$ and specificity of $70 \% \%^{2}$. Amyloid deposition can be readily identified by Congo red staining of carpal tunnel biopsies taken at routine decompression surgery. Immunohistochemistry $(\mathrm{IHC})$ is usually able to determine the amyloid

A

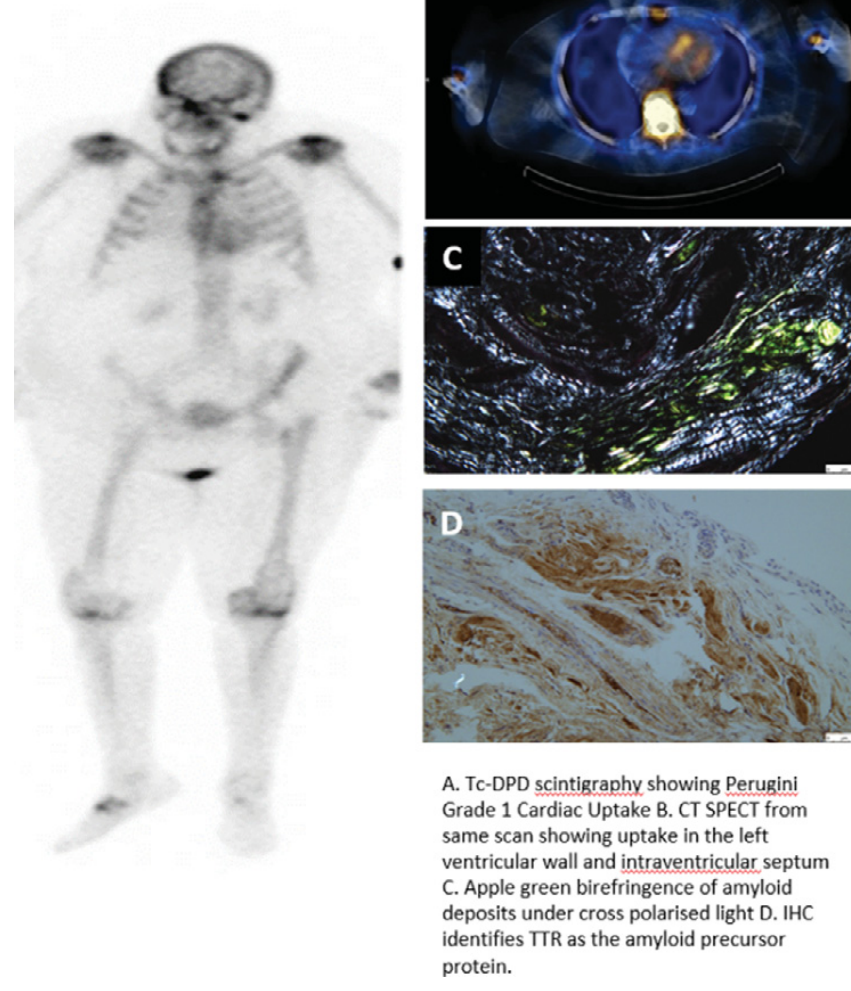

fibril protein (amyloid type). Rheumatologists are ideally placed to request CT biopsy. Developing this underused but diagnostic test is of particular importance in light of emerging therapies for ATTR amyloidosis.

Objectives: To determine the utility of CT biopsy as a diagnostic tool in systemic ATTR amyloidosis, and its utility in combination with Tc-DPD scintigraphy in identifying pre-clinical cardiac amyloidosis.

Methods: CT biopsies were taken at decompression surgery. Biopsies were stained with Congo red and viewed under cross polarised light. IHC was used to type amyloid deposits using standardised techniques.

Results: We analysed 37 CT biopsies, $73 \%$ female, median age 65.8 yrs (35-87 yrs). 5 biopsies contained amyloid deposits (13.5\%) by Congo red staining. 3 were typed as ATTR amyloid using immunohistochemistry (8\%), median age 80.35 yrs. The amyloid type could not be determined by IHC in two cases. No cases of proven ATTR amyloid had a history of heart failure symptoms. Of these, a $74 \mathrm{yr}$ old male attended the NAC for diagnostic work up. He had a normal ECG and Echocardiogram, however, Tc-DPD scintigraphy was able to demonstrate low grade uptake.

Conclusions: Carpal tunnel biopsy can readily identify ATTR amyloid deposition and may identify those at risk of developing cardiac ATTR amyloidosis in the future, permitting earlier intervention with novel therapeutics aimed at preventing accumulation of amyloid. This ongoing study aims to identify the UK prevalence of ATTR amyloid in those with carpal tunnel syndrome and to create a cohort of those who may develop systemic ATTR amyloidosis to further elucidate the disease natural history.

References:

[1] Senile systemic amyloidosis: clinical features at presentation and outcome. Pinney JH et al., J Am Heart Assoc. 2013 Apr 22;2(2). doi: 10.1161/ JAHA.113.000098.

[2] Nonbiopsy Diagnosis of Cardiac Transthyretin Amyloidosis. Gillmore JD et al., Circulation. 2016 Jun 14:133(24):2404-12. doi: 10.1161/CIRCULATIONAHA.116.021612

Disclosure of Interest: None declared

DOI: 10.1136/annrheumdis-2017-eular.5040

\section{AB1065 NEEDLE VERSUS FORCEPS TECHNIQUE IN ULTRASOUND-GUIDED SYNOVIAL BIOPSY OF THE KNEE JOINT}

T. Hügle ${ }^{1}$, P. Urbaniak ${ }^{1}$, M. Müller-Gerbl ${ }^{2}$, C. Marx ${ }^{3}$, G. Tamborrini ${ }^{3}$. ${ }^{1}$ Department of Rheumatology, University Hospital Basel; ${ }^{2}$ Institute of Anatomy, University Basel; ${ }^{3}$ Ultrasound Center Rheumatology, Basel, Switzerland

Background: Ultrasound-guided synovial biopsy is increasingly applied in rheumatology. Usually forceps- or needle-based techniques are used. So far there has been no direct comparison of different devices regarding their suitability in high resolution musculoskeletal ultrasound (hrMSUS)-guided synovial biopsy.
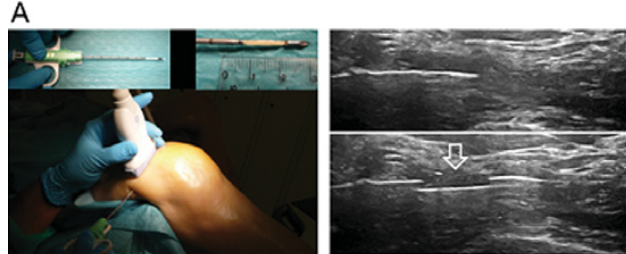

B
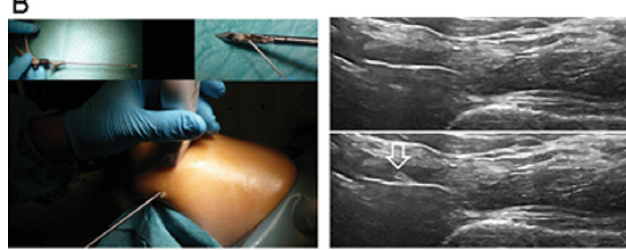

C
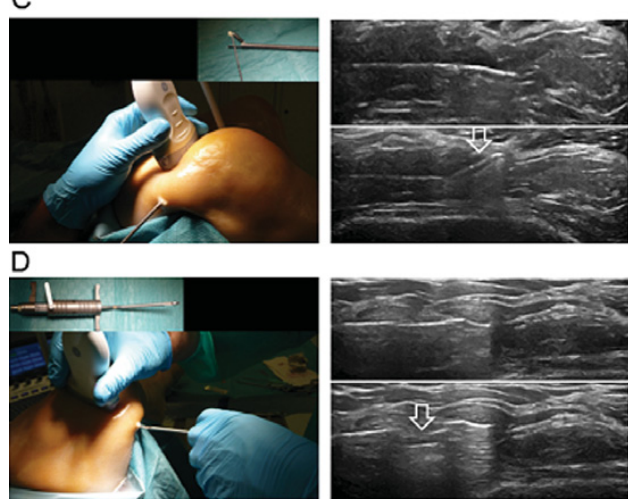
Objectives: To compare different forceps and needle-based instruments in hrMSUS-guided synovial biopsy in a cadaver study.

Methods: A core needle biopsy (A, Quickcore, Cook Medical, Bloomington, IN, USA), a retrograde forceps (B, Retroforce, Karl-Storz GmbH Tuttlingen, Germany), an anterograde arthroscopy forceps (C, Karl Storz GmbH, Tuttlingen, Germany) and an convexly shaped integrated core needle system (D, Synovex, Hipp Medical, Kolbingen, Germany) were tested for ultrasound-guided synovial biopsy of the suprapatellar recess in cadaver knee joints. Four senior rheumatologists scored each intervention from $0-5$ regarding the following characteristics: visualization, handiness, accuracy, synovial tissue yield, invasiveness and overall suitability. Each intervention was recorded as static images and video clips.

Results: In all devices, enough representative synovial tissue was obtained and the instruments were all well visualized by hrMSUS. Core needle biopsy and the integrated needle system were best visualized due to their horizontally shaped closing mechanism. The core needle obtained a high yield of superficial synovial tissue and was the least invasive procedure. Despite handiness and accuracy were higher in the forceps instruments, overall suitability for hrMSUS-guided synovial biopsy was rated highest for the core biopsy needle.

Conclusions: Technically, all of the tested devices can be used for hrMSUSguided synovial biopsy. Core needle biopsy seems to be most suitable for this intervention due to a low invasiveness, good visualisation and optimal yield of superficial synovial tissue.

\section{References:}

[1] Hügle $T$ et al. Retrograde synovial biopsy of the knee joint using a novel biopsy forceps. Arthrosc Tech. 2014;3(3):e317-9.

[2] Hügle T et al. Development of a New Device for Synovial Biopsies. Surg Innov. 2015;22(5):496-9.

Disclosure of Interest: T. Hügle Shareholder of: Prof. Hügle contributed to the development of the Retroforce and Synovex instruments and receives royalties from Karl-Storz GmbH and Hipp Medical AG., P. Urbaniak: None declared, M. Müller-Gerbl: None declared, C. Marx: None declared, G. Tamborrini: None declared

DOI: 10.1136/annrheumdis-2017-eular.5933

\section{AB1066 DIAGNOSTIC UTILITY OF THE MEDIAN/ULNAR NERVE CROSS-SECTIONAL AREA RATIO IN CARPAL TUNNEL SYNDROME}

T. Atan ${ }^{1}$, Z. Günendi ${ }^{2} .{ }^{1}$ Physical Medicine and Rehabilitation, Hitit University, Çorum Training and Research Hospital, Çorum; ${ }^{2}$ Physical Medicine and Rehabilitation, Gazi University Faculty of Medicine, Ankara, Turkey

Background: The most commonly used ultrasonographic measurements for the diagnosis of CTS are measurement of the median nerve cross-sectional area $(m-C S A)$ at different levels of the carpal tunnel. ${ }^{1}$ The cross-sectional area of a nerve may differ according to biometric characteristics such as age, sex, height, weight and wrist thickness. ${ }^{2-4}$

Objectives: The aim of this study was to assess the diagnostic utility of the ultrasonographic ratio of $\mathrm{m}$-CSA to ulnar nerve cross-sectional area (u-CSA), the m-CSA/ u-CSA ratio, in carpal tunnel syndrome (CTS).

Methods: Patients $(n=50)$ with positive symptoms and electromyography results of CTS and control subjects $(n=50)$ with negative electromyography results of CTS were evaluated. The most symptomatic hand of each participant were included in the assesment. Ultrasonographic m-CSA and u-CSA measurements were made at the level of the pisiform bone, and m-CSA/u-CSA ratio was calculated.

Results: Using the m-CSA cut-off value of $9.95 \mathrm{~mm}^{2}$ showed a sensitivity of $92 \%$ and a specificity of $42 \%$. Conversely, the cut-off value $13.90 \mathrm{~mm}^{2}$ showed a sensitivity of $56 \%$ and a specificity of $92 \%$. Using the cut-off value 2.96 for the ratio of m-CSA/u-CSA showed a sensitivity of $86 \%$ and a specificity of $38 \%$ while using the cut-off value 3.71 showed a sensitivity of $52 \%$ and a specificity of $90 \%$ in the diagnosis of CTS.

Conclusions: The ratio of $\mathrm{m}-\mathrm{CSA} / \mathrm{u}-\mathrm{CSA}$ at the level of the pisiform bone did not provide an additional benefit for the diagnosis of CTS. Ultrasonographic $\mathrm{m}$-CSA measured at the same level was found to be more sensitive and specific method. References:

[1] Mondelli M, Flippou G, Gallo A, Frediani B. Diagnostic utility of ultrasonography versus nerve conduction studies in mild carpal tunnel syndrome. Arthritis Rheum. 2008;59:357-66.

[2] Bathala L, Kumar P, Kumar K, Shaik A, Visser LH. Normal values of median nerve cross-sectional area obtained by ultrasound along its course in the arm with electrophysiological correlations, in 100 Asian subjects. Muscle Nerve. 2014;49:284-6.

[3] Qrimli M, Ebadi H, Breiner A, et al. Reference values for ultrasonograpy of peripheral nerves. Muscle Nerve. 2016;53:538-44.

[4] Won SJ, Kim BJ, Park KS, Yoon JS, Choi H. Reference values for nerve ultrasonography in the upper extremity. Muscle Nerve. 2013;47:864-71.

Disclosure of Interest: None declared

DOI: 10.1136/annrheumdis-2017-eular.6550

\section{AB1067 SUITABILITY OF CADAVER MODELS IN ULTRASOUND DIAGNOSTICS AND INTERVENTIONS IN RHEUMATOLOGY: FOOT AND ANKLE}

V. Halász ${ }^{1}$, Á. Nemeskéri ${ }^{1}$, D. Bong ${ }^{2}$, P. Mandl ${ }^{3}$, I. Möller ${ }^{2}$, E. Naredo ${ }^{4}$, P.V. Bálint ${ }^{5}$ on behalf of EULAR Anatomy for the Image Study Group. ${ }^{1}$ Department of Anatomy, Histology and Embriology, Semmelweis University, Budapest, Hungary; ${ }^{2}$ Department of Rheumatology, Instituto Poal de Reumatologia, Barcelona, Spain; ${ }^{3}$ Division of Rheumatology, Medical University of Vienna, Vienna, Austria; ${ }^{4}$ Department of Rheumatology, Hospital Universitario Fundación Jiménez Díaz and Autónoma University, Madrid, Spain; ${ }^{5} 3 r d$ Department of Rheumatology, National Institute of Rheumatology and Physiotherapy, Budapest, Hungary

Background: Employment of cadaver specimens in ultrasonography provides a useful and safe model for education, enhances the anatomical knowledge of sonographers and may help determine the accuracy of ultrasound-guided interventions. Objectives: In this systemic literature review (SLR) we assessed the role and use of cadaver specimens in sonographic studies of the foot and ankle in the field of rheumatology.

Methods: For our literature review we utilized the MEDLINE database, which were supplemented by searches in Google Scholar and Science Direct when the articles were not available through PubMed. Original studies in English language were included in the full paper review with an exception of three German language studies with English abstracts were also included. In the full paper review studies were selected for inclusion featured the sonographic study of cadaver specimens of the foot and ankle. Data were extracted on study characteristics and interventions.

Results: The search yielded 1241 articles, of which 130 were selected for detailed review. In the end, 23 full papers met inclusion criteria. The studies could be grouped as follows: description of detailed ultrasound anatomy (9), testing of accuracy of ultrasound guided interventional procedures (8), examination of artificial tears and lesions (4), foreign bodies (1) and joint effusions (1). The results that were obtained in the studies of the fully reviewed papers utilized a total of 294 cadaveric specimens, with an average of 12.78 (range: 1-48) cadaveric specimens included in each study.

Conclusions: The use of cadaver specimens of the foot and ankle may facilitate the validation of new sonographic methods which assess these joint regions, however the major disadvantage of these studies was the low number of cadaveric specimens.

Disclosure of Interest: None declared

DOI: 10.1136/annrheumdis-2017-eular.4635

\section{AB1068 ULTRASOUND OF SALIVARY GLANDS IN SJOGREN'S SYNDROME- WHICH SEMI-QUANTITATIVE SCORING SYSTEM IS THE BEST?}

V.C. Iorgoveanu ${ }^{1}$, V. Bojinca ${ }^{2}$, V. Vlad ${ }^{3}$, D. Mazilu ${ }^{4}$, I. Saulescu ${ }^{2}$, L. Groseanu ${ }^{2}$ D. Opris ${ }^{2}$, A. Balanescu ${ }^{2}$, C. Constantinescu ${ }^{2}$, D. Predeteanu ${ }^{2}$, R. Ionescu ${ }^{2}$.

${ }^{1}$ Rheumatology, Carol Davila University of Medicine and Pharmacy;

${ }^{2}$ Rheumatology, Saint Mary Clinical Hospital, UMF Carol Davila; ${ }^{3}$ Rheumatology, Saint Mary Clinical Hospital; ${ }^{4}$ Saint Mary Clinical Hospital, UMF Carol Davila,

Bucharest, Romania

Background: Sjogren Syndrome (SS) affects mainly exocrine glands. The latest diagnostic criteria designed for clinical studies are also used as guidance in clinical practice [1].

Ultrasonography (US) demonstrates specificity and sensibility in parotid and submandibular gland evaluation (SG). Parameters considered are echogenicity, homogeneity and margins regularity $[1,2,3]$. To standardize the assessment of $B$ mode US of SG, different semi-quantitative scores were proposed.

Objectives: To apply and compare 9 US semi-quatitative scoring systems in B mode scanning of salivary glands in Sjogren Syndrome.

Methods: A research using keywords "salivary glands", "ultrasonograpy", "Sjogren Syndrome", "semi-quantitative score" in Medline/Pubmed was performed. There was a selection of most relevant articles. There were not considered relevant publications with impact factor $<1$. We performed the examination on $S G$ in $B$ mode US and applied these scores (De Vita, Niemela, Hocevar, Salaffi, Yukinori, Cornec, Theander) to our patients (primary and secondary SS).

Results: Eighty four $S G$ in patients diagnosed with primary and secondary $(57.15 \%)$ SS were assessed. In the group of patients with SSA/SSB presence $(85.7 \%)$, mean score was De Vita 1.78+/-1.21, Niemela 2.56+/-2.17, Hocevar and Wernicke 2.39+/-2.14, Salaffi 2.83+/-2.52, Yukinori 2.39+/-2.14, Milic 3.39+/-2.14, Cornec $1.78+/-1.215$, Theander $1.28+/-0.752$. Schirmer test and the need for using the artificial tears was correlated to $S G$ alterations in scoring systems proposed by Niemela $(r 0.465, p<0.05)$ and Salaffi $(r 0.496, p<0.02)$. All scoring systems were strongly correlated between them $(r>0.8, p<0.01)$.

Conclusions: Inhomogeneity of parenchyma was considered in all scoring systems. Others considered relevant glandular dimension and margins regularity $[2,3.4]$. There was no difference between the scoring systems. Xeroftalmia valided through Schirmer test is correlated to SG parenchymal alterations. Our data is an update about semi-quantitative scoring systems in US of SG in SS.

References:

[1] Vitali C, Bombardieri S, Jonsson R et al. Classification criteria for Sjögren's 